VOL. 65 (2002) [449-460]

\title{
COMPLETELY MONOTONE FADING MEMORY RELAXATION MODULII
}

\section{R.S. ANDERSSEN AND R.J. LOY}

In linear viscoelasticity, the fundamental model is the Boltzmann causal integral equation

$$
\sigma(t)=\int_{-\infty}^{t} G(t-\tau) \dot{\gamma}(\tau) d \tau
$$

which defines how the stress $\sigma(t)$ at time $t$ depends on the earlier history of the shear rate $\dot{\gamma}(\tau)$ via the relaxation modulus (kernel) $G(t)$. Physical reality is achieved by requiring that the form of the relaxation modulus $G(t)$ gives the Boltzmann equation fading memory, so that changes in the distant past have less effect now than the same changes in the more recent past. A popular choice, though others have previously been proposed and investigated, is the assumption that $G(t)$ be a completely monotone function. This assumption has much deeper ramifications than have been identified, discussed or exploited in the rheological literature. The purpose of this paper is to review the key mathematical properties of completely monotone functions, and to illustrate how these properties impact on the theory and application of linear viscoelasticity and polymer dynamics. A more general representation of a completely monotone function, known in the mathematical literature, but not the rheological, is formulated and discussed. This representation is used to derive new rheological relationships. In particular, explicit inversion formulas are derived for the relationships that are obtained when the relaxation spectrum model and a mixing rule are linked through a common relaxation modulus.

\section{INTRODUCTION}

The stress-strain behaviour of a material, such as a synthetic or a manufactured polymer (for example, plastics, rubbers and food doughs) or a naturally occurring biopolymer (for example, wood, bone and muscle), is modelled using a constitutive relationship. Because of the complex ways in which many materials respond when deformed, the formulation of constitutive relationships involves quite deep and challenging mathematical manipulations (see [12]).

Received 24th October, 01

The authors thank the referee for a very careful reading of the paper which resulted in the clarification of several points.

Copyright Clearance Centre, Inc. Serial-fee code: 0004-9727/02 \$A2.00+0.00. 
For a linear viscoelastic material, such as a synthetic polymer subjected to small deformations, the Boltzmann causal integral equation

$$
\sigma(t)=\int_{-\infty}^{t} G(t-\tau) \dot{\gamma}(\tau) d \tau
$$

is the accepted constitutive relationship. It models how, in such materials, the stress $\sigma(t)$, at time $t$, depends on the earlier history of the shear rate $\dot{\gamma}(\tau)$. For a given material, the essence of its viscoelasticity is encapsulated in the form and structure of the relaxation modulus $G(t)$. On physical grounds, this kernel cannot be an arbitrary function. In particular, through the choice of $G(t)$, equation (1) must be such that changes in the shear rate in the distant past must have less effect now than the same changes in the more recent past. The regularity imposed on $G(t)$ to achieve this is called fading memory.

Various definitions for the concept of fading memory have been proposed and investigated (for example, $[\mathbf{1}, \mathbf{9}]$ ). A popular choice $([\mathbf{7}, \mathbf{5}]$ ) is to assume that $G(t)$ is completely monotone $([\mathbf{1 0}, \mathbf{1 8}])$; namely,

$$
(-1)^{n} \frac{d^{n} G(t)}{d t^{n}} \geqslant 0, \quad n \geqslant 0, \quad t \geqslant 0 .
$$

Rheologically, the popularity of this definition relates directly to the fact that $G(t)$ can be characterised in terms of the concept of a relaxation spectrum $H(\tau)$ by

$$
G(t)=\int_{0}^{\infty} \exp (-t / \tau) \frac{H(\tau)}{\tau} d \tau, \quad H(\tau) \geqslant 0, \quad t \geqslant 0 .
$$

Such $G(t)$ are automatically completely monotone, and the simple change of variable $s=1 / \tau$ yields

$$
G(t)=\int_{0}^{\infty} \exp (-t s) \frac{H(1 / s)}{s} d s
$$

which fits the Laplace transform definition for completely monotone functions ([18, p.160]). As noted below, it follows from this result that any completely monotone function is the restriction to the positive half-line $[0, \infty)$ of a function analytic in the right half plane.

Pragmatically, the importance of the choice of complete monotonicity as the definition of fading memory is that one only needs to derive a positive estimate of $H(\tau)$, from given rheological measurements, in order to guarantee that $G(t)$, determined by (3), is completely monotone. In addition, equation (3) yields a formal characterisation of the concept of a relaxation spectrum $H(\tau)$ of a polymer in terms of the kernel $G(t)$.

The paper has been organised in the following manner. The relevant properties of completely monotone functions are reviewed, and, where appropriate, derived in Section 2. The equivalencing of the relaxation spectrum and mixing rule representations of $G(t)$ is examined in Section 3. Explicit inversion formulas are constructed in Section 4 for the resulting relationships. These results extend and complement similar results derived by $[16,17]$. 


\section{Mathematical Properties of Completely Monotone fading Memory FUNCTIONS}

The need to guarantee that equation (1) induces fading memory, in terms of how the stress responds to different shear rates, was first recognised by Boltzmann [6]. A popular choice, for both practical and theoretical reasons, is to define $G(t)$ to have fading memory, if it is completely monotone. We make the following definition.

Definition 1: A function $G(t)$ on $[0, \infty)$ has fading memory, if and only if it has the form

$$
G(t)=\int_{0}^{\infty} \exp (-t / \tau) d \mu(\tau), \quad t \geqslant 0
$$

for some positive finite measure $\mu$ on $[0, \infty)$. Equivalently, by introducing the change of variable $s=1 / \tau$,

$$
G(t)=\int_{0}^{\infty} \exp (-t s) d \nu(s)
$$

where $d \nu(s)=s^{-2} d \mu\left(s^{-1}\right)$.

Let $M^{+}[0, \infty)$ denote the wedge of finite positive Borel measures on $[0, \infty)$ under convolution product, and $\mathcal{F}$ the set of completely monotone functions on $[0, \infty)$.

Proposition 1. The Laplace transform (6) is an isomorphism from $M^{+}[0, \infty)$ onto $\mathcal{F}$ taken with pointwise product.

Proof: It is standard that the Laplace transform is an isomorphism from $M^{+}[0, \infty)$ into $\mathcal{F}$. Bernstein's theorem ([18, Theorem 12a, p.160]) establishes that it is surjective. $]$

It is an immediate consequence of this that any completely monotone function is the restriction to the positive half-line $[0, \infty)$ of a function analytic in the right half plane. It is this fact which gives the concept of complete monotonicity its specialised structure, and explains why the definition (2) is not as innocuous as it initially appears. For example, a non-constant completely monotone function must be strictly decreasing on $[0, \infty)-$ otherwise it will be constant on some interval, and hence everywhere by analyticity. We also emphasise that $\mathcal{F}$ is a wedge; that is, it is closed under positive linear combinations and products. That it is closed under products can also be established directly. For supposing $G_{1}$ and $G_{2}$ have fading memory, Leibnitz's formula yields

$$
(-1)^{n} \frac{d^{n}\left(G_{1} G_{2}\right)(t)}{d t^{n}}=\sum_{i+j=n}(-1)^{i} \frac{d^{i}\left(G_{1}\right)(t)}{d t^{i}} \cdot(-1)^{j} \frac{d^{j}\left(G_{2}\right)(t)}{d t^{j}} \geqslant 0 .
$$

One result, that can be found in the mathematics literature $([11])$, but has not yet been exploited in the study of the polymer dynamics of linear viscoelastic materials having completely monotone fading memories, is given by 
Lemma . Let $f:[0, \infty) \rightarrow[0, \infty)$ be strictly monotonically increasing and infinitely differentiable, with $f(0)>0$. For the function

$$
G(t)=\int_{0}^{\infty} \exp (-f(t) / \tau) d \mu(\tau)
$$

to have fading memory, it is sufficient that $f^{\prime}(t)$ has fading memory.

Proof: Suppose that $t>0$. Then the $n$-th derivative of the integrand with respect to $t$ is a sum of terms of the form $\tau^{-m} h_{m}(t) \exp (-f(t) / \tau)$ for $1 \leqslant m \leqslant n$, for some functions $h_{m}$. Since $f(t)>0$, this is bounded as $\tau \rightarrow 0$, hence integrable with respect to $\mu$. It follows that differentiation through the integral is allowable. The same reasoning (using $f(0)>0$ ) shows that for each $n \geqslant 0, \lim _{t \downarrow 0} G^{(n)}(t)$ exists. It follows that $G$ is infinitely differentiable on $[0, \infty)$ with the expected derivative.

Let

$$
\phi(x)=\int_{0}^{\infty} \exp (-x / \tau) d \mu(\tau), \quad x \geqslant 0
$$

Then,

$$
G(t)=(\phi \circ f)(t)
$$

where the symbol "o" denotes composition. Thus, sufficiency follows from [10, Criterion 2 (p.441)], where it is established that such compositions are completely monotone.

The hypothesis that $f(0)>0$ was only required to ensure suitable behaviour of the differentiated integrand near $\tau=0$. In rheological applications, because one does not have infinitely small molecular weights, the support of $\mu$ will not contain zero. In such situations, the extra hypothesis is no longer required. Indeed, without loss of generality, in order to ensure that $f(0)=0$, one can apply the transformation

$$
\exp (-f(t) / \tau)=\exp (-[f(t)-f(0)] / \tau) \exp (-f(0) / \tau)
$$

where the factor $\exp (-f(0) / \tau)$ can be asborbed into the definition of $\mu(\tau)$ to give a new measure that dies off sufficiently rapidly near $\tau=0$ to guarantee that differentiation through the integral sign remains valid.

As an immediate consequence of the fact that $\mathcal{F}$ is a wedge, any non-negative integer power of a fading memory function will have fading memory. On the other hand, a fractional power of a fading memory function does not necessarily have fading memory. Indeed, if it is assumed that $F(t)$ has fading memory, then

$$
(-1)^{n} \frac{d^{n} F}{d t^{n}}(t) \geqslant 0
$$

for all non-negative integers $n$, and for all $t \geqslant 0$. Consider $F_{r}(t)=[F(t)]^{r}$, where $0<r<1$. Then

$$
(-1) \frac{d F_{r}(t)}{d t}=r F_{r-1}(t) \frac{d F(t)}{d t} \geqslant 0
$$


and

$$
\frac{d^{2} F_{r}(t)}{d t^{2}}=r(r-1) F_{r-2}(t)\left[\frac{d F(t)}{d t}\right]^{2}+r F_{r-1}(t) \frac{d^{2} F(t)}{d t^{2}}
$$

Because $r<1$, the first term on the right hand side of this last equation is negative while the second is positive. Consequently, the conditions for completely monotonocity fail for the second derivative of $F_{r}(t)$ for any $F(t)$ for which $d F / d t(0) \neq 0$ and $d^{2} F / d t^{2}(0)=0$.

As is implicit in this simple example, the fractional power of a completely monotone function will only be completely monotone under special circumstances. In fact, all fractional powers of a completely monotone function will be completely monotone, if attention is limited to the very special subset of completely monotone functions that have infinitely divisible measures (see [10]). If the fading memory of $G(t)$ is generated by an infinitely divisible measure, then $([10$, p.450]) there is a function $f:[0, \infty) \rightarrow[0, \infty)$ such that

$$
G(t)=G(0) \exp (-f(t)), \quad f(0)=0, \quad \frac{d f}{d t} \in \mathcal{F}
$$

In terms of the above Lemma, this corresponds to the measure being a point mass. More generally, an infinitely divisible measure is either a point mass or has unbounded support ([10, p.177]).

NotE. The above discussion relates to functions defined on the half-line (an assumption implicit in the discussion about infinite divisibility in [11]). The normal (Gaussian) distribution is infinitely divisible on the whole line, but does not satisfy the above condition of equation (11).

Another consequence of the wedge property of $\mathcal{F}$, that will be exploited below, is that one can start with a subfamily $G_{r}(t), r=1,2, \ldots, N$, of functions in $\mathcal{F}$ and build new elements $G(t) \in \mathcal{F}$ such as

$$
G(t)=\sum_{r=1}^{N} W_{r}\left[G_{r}(t)\right]^{r}, \quad W_{r} \geqslant 0 .
$$

It follows from the above results that, for a given $G(t) \in \mathcal{F}$, there are various ways in which it could be constructed to take the form (7) including

$$
G_{r}(t)=\int_{0}^{\infty} \exp \left(-g_{r}(t) / \tau\right) \frac{H_{r}(\tau)}{\tau} d \tau, \quad g_{r}(t) \geqslant 0, \quad \frac{d g_{r}(t)}{d t} \in \mathcal{F}, \quad H_{r}(\tau) \geqslant 0 .
$$

Though, at first sight, this appears to be a quite innocuous result, it has major ramifications for the modelling of the dynamics of a linear viscoelastic material, if it can be assumed that the relaxation modulus of that material $G(t) \in \mathcal{F}$. In particular, this result suggests that the dynamics of a polymer may be decomposable into a linear combination of more fundamental dynamics. The actual characterisation of these more fundamental dynamics becomes a rheological matter that will be discussed below and pursued independently. 


\section{Equivalencing of Relaxation Spectrum and Mixing Rule REPRESENTATIONS FOR $G(t)$}

It is clear from the rheological literature that, for much industrial decision-making, the molecular weight distribution $w(m)$ of a polymer represents a suitable and adequate molecular characterisation $([\mathbf{1 4}, \mathbf{1 5}])$. Consequently, there is great interest in its determination. A wide variety of methods, ranging from the direct experimental (HPLC, size exclusion and triple $F$ ) to the inversion of indirect rheological measurement, are available and applied on a regular basis ([4]). Though the direct methods are quite appropriate in the study of the structure of DNA and proteins, it is the inversion of rheological measurements which are normally applied in the study of synthetic polymers.

Of these methods, the most natural and popular are mixing rules which take the form

$$
G(t)=G_{N}^{0}\left[\int_{0}^{\infty} k(t, m) w(m) d m\right]^{\gamma}, \quad G_{N}^{0}=\text { constant }
$$

where $G(t)$ denotes the relaxation modulus, $w(m)$ the molecular weight distribution of the polymer, and $\gamma$ some real value which reflects the nature of the polymer dynamics under consideration $([\mathbf{2}, \mathbf{3}, \mathbf{1 6}, \mathbf{1 7}])$. The classical choices are $\gamma=1$ and $\gamma=2$, which correspond, respectively, to single and double reptation. The kernel $k(t, m)$ is chosen to reflect how the various components of $\dot{w}(m)$ contribute to $G(t)$. There is an extensive literature on the subject, see also $[15,13]$.

On the other hand, a completely monotone relaxation modulus $G(t)$ has a relaxation spectrum $H(\tau)$ characterisation given by equation (3). In [16], the idea has been exploited of using $G(t)$ as the link concept which allows one to construct the following types of relationship between $w(m)$ and $H(\tau)$

$$
\int_{0}^{\infty} \exp (-t / \tau) \frac{H(\tau)}{\tau} d \tau=G_{N}^{0}\left[\int_{0}^{\infty} k(t, m) w(m) d m\right]^{\gamma}, \quad \tau=\tau(m),
$$

where the dependence of the relaxation time $\tau$ on the molecular weight $m$ must be made explicit in order to establish the coupling between both sides of this equation. This equivalencing has allowed $[16,17]$ to derive various analytic expressions between $w(m)$ and $H(\tau)$ and to put the study of mixing rules on a more rigorous footing.

However, this equivalencing imposes strong constraints on the structure of the kernel $k(t, m)$ and the value of the exponent $\gamma$. In particular, consistency demands that, as for $G(t)$, the right hand side of equation (15) must have a form that guarantees its complete monotonicity. If $\gamma$ is constrained to be an integer $r$, then, on the basis of the Lemma and the related discussion, a general form that the mixing rule counterpart of $G_{r}(t)$ of (13) 
could have is

$$
\begin{aligned}
G_{r}(t) & =G_{N}^{0}\left[\int_{0}^{\infty} k_{r}(m) \exp \left(-f_{r}(t) / \tau_{r}(m)\right) w_{r}(m) d m\right]^{r}, \\
k_{r}(m) & \geqslant 0, \quad w_{r}(m) \geqslant 0, \\
\frac{d f_{r}}{d t} & \in \mathcal{F}, \quad \tau_{r}(m) \geqslant 0 .
\end{aligned}
$$

This leads naturally to the following general form for a mixing rule proposed by [2]

$$
G(t)=G_{N}^{0} \sum_{r=1}^{N} W_{r}\left[\int_{0}^{\infty} k_{r}(m) \exp \left(-f_{r}(t) / \tau_{r}(m)\right) w_{r}(m) d m\right]^{r}, \quad k_{\tau}(m) \geqslant 0 .
$$

On the other hand, for a given $\tau=\tau_{r}(m)$, the functions $G_{r}(t)$ of equation (13) can be rewritten in terms of functions of $m$ with the following form

$$
\begin{aligned}
G_{r}(t) & =\int_{0}^{\infty} \frac{\bar{H}_{r}(m) \tau_{r}^{\prime}(m)}{\tau_{r}(m)} \exp \left(-g_{r}(t) / \tau_{r}(m)\right) d m, \\
\bar{H}_{\tau}(m) & =H_{r}(\tau(m)), \\
\tau_{r}^{\prime}(m) & =\frac{d \tau_{r}(m)}{d m} .
\end{aligned}
$$

On equating these last two expressions for $G_{r}(t)$, one obtains

$$
\begin{aligned}
\int_{0}^{\infty} \frac{\bar{H}_{r}(m) \tau_{r}^{\prime}(m)}{\tau_{r}(m)} \exp \left(-g_{r}(t) / \tau_{r}(m)\right) d m \\
=G_{N}^{0}\left[\int_{0}^{\infty} k_{r}(m) \exp \left(-f_{r}(t) / \tau_{r}(m)\right) w_{r}(m) d m\right]^{r}
\end{aligned}
$$

The importance of this last expression is that it yields an equivalencing between the components of a decomposition of the relaxation spectrum of a polymer and the molecular weight distribution contributing to those components.

\section{Construction of Inversion Formulas}

Using special cases of the above equivalencing (19), various authors, including [16, 17] and [19], have derived explicit inversion formulas for the molecular weight distribution as a function of the relaxation spectrum and vice versa. The formulas derived by $[16,17]$ exploit the fact that the $\beta$ power of any definite integral $\int_{a}^{b} f(x) d x$ has the following decompositions

$$
-\int_{a}^{b} \frac{d}{d x}\left[\int_{x}^{b} f(\bar{x}) d \bar{x}\right]^{\beta} d x=\left[\int_{a}^{b} f(\bar{x}) d \bar{x}\right]^{\beta}=\beta \int_{a}^{b} f(x)\left[\int_{x}^{b} f(\bar{x}) d \bar{x}\right]^{\beta-1} d x
$$

However, in this earlier work, it was not explicitly assumed that $\beta$ was an integer. A key advantage of the new mixing rule (17) is that the values of $r$ are constrained to be integers. Taking this into account allows one to construct the following inversion formulas from equation (19). 
SINGLE REPTATION. When $r=1$, which corresponds to classical single reptation dynamics, equation (19) can be reorganised to yield

$$
\begin{aligned}
\int_{0}^{\infty} \exp & \left(-g_{1}(t) / \tau_{1}(m)\right) \\
& {\left[\frac{\bar{H}_{1}(m) \tau_{1}^{\prime}(m)}{\tau_{1}(m)}-G_{N}^{0} k_{1}(m) w_{1}(m) \exp \left(-\left(f_{1}(t)-g_{1}(t)\right) / \tau_{1}(m)\right)\right] d m=0 . }
\end{aligned}
$$

For given $\bar{H}_{1}(m), \tau_{1}(m), g_{1}(t)$ and $f_{1}(t)$ and non-zero $k_{1}(m)$, this expression uniquely determines $w_{1}(m)$. For suppose that $w_{1}(m)$ and $w_{*}(m)$ both satisfy $(21)$. Subtraction yields

$$
\int_{0}^{\infty} k_{1}(m) \exp \left(-f_{1}(t) / \tau_{1}(m)\right)\left[\dot{w}_{1}(m)-w_{*}(m)\right] d m=0, \quad t \geqslant 0
$$

On the one hand, the function

$$
F(z)=\int_{0}^{\infty} k_{1}(m) \exp \left(-z / \tau_{1}(m)\right)\left[w_{1}(m)-w_{*}(m)\right] d m,
$$

is an analytic function of $z$ defined on $\operatorname{Re} z>0$. Equation (22) asserts that this analytic function is zero on the non-trivial interval $\left\{f_{1}(t), t \geqslant 0\right\}$. Since this interval includes an infinity of points in the complex plane with a limit point, it follows from a standard result in complex variable theory that $F(z)$ is identically zero for $\operatorname{Re} z \geqslant 0$; and, in particular, $F(t)=0$ for all real values of $t \geqslant 0$.

Introducing the change of variable $p=1 / \tau_{1}(m)$ into (23), with $m=\phi_{1}(p)$ denoting the inverse relation, yields

$$
\int_{0}^{\infty} \tilde{k}_{1}(p) \exp (-t p) \frac{\tilde{w}_{1}(p)-\tilde{w}_{*}(p)}{p^{2} \widetilde{\tau}_{1}^{\prime}(p)} d p=0, \quad t \geqslant 0,
$$

where $\tilde{k}_{1}(p)=k_{1}\left(\phi_{1}(p)\right), \tilde{\tau}_{1}(p)=\tau_{1}\left(\phi_{1}(p)\right), \widetilde{w}_{1}(p)=w_{1}\left(\phi_{1}(p)\right)$ and $\tilde{w}_{*}(p)=w_{*}\left(\phi_{1}(p)\right)$. The invertibility of the Laplace transform shows that $\widetilde{w}_{1}(p)=\widetilde{w}_{*}(p)$, whence $w_{1}(m)$ $=w_{*}(m)$, which establishes the required uniqueness.

When $f_{\mathrm{i}}(t)=g_{1}(t)$ for all $t \geqslant 0,(21)$ gives

$$
\int_{0}^{\infty} \exp \left(-g_{1}(t) / \tau_{1}(m)\right)\left[\frac{\bar{H}_{1}(m) \tau_{1}^{\prime}(m)}{\tau_{1}(m)}-G_{N}^{0} k_{1}(m) w_{1}(m)\right] d m=0 .
$$

NotE. This result will also hold under the (apparently) weaker hypothesis that $f_{1}(s)$ $=g_{1}(s)$ for an infinite set of points $s$ which is not too sparse (see [10, Chapter XIII, Section 1, p.430]).

On applying the same argument used above to prove that $w_{1}(m)=w_{*}(m)$, it follows that

$$
\frac{\bar{H}_{1}(m) \tau_{1}^{\prime}(m)}{\tau_{1}(m)}=G_{N}^{0} k_{1}(m) w_{1}(m)
$$


is the only expression which relates $w_{1}(m)$ to $\bar{H}_{1}(m), \tau_{1}(m)$ and $k_{1}(m)$.

We-remark that it is easily demonstrated that there are solutions to (21) when $f_{1}(t) \neq g_{1}(t)$. Simply consider (19) with $r=1, k_{1}(m)=1, f_{1}(t)=t$. Setting $p=1 / \tau_{1}(m)$, one obtains, similar to equation (25),

$$
\int_{0}^{\infty} \exp (-t p) \frac{\widetilde{w}_{1}(p)}{p^{2} \widetilde{\tau}_{1}^{\prime}(p)} d p=\int_{0}^{\infty} \frac{\widetilde{H}_{1}(p)}{p} \exp \left(-g_{1}(t) p\right) d p
$$

where $\widetilde{H}_{1}(p)=\bar{H}_{1}\left(\phi_{1}(p)\right), \widetilde{w}_{1}(p)=w_{1}\left(\phi_{1}(p)\right)$, and $\widetilde{\tau}_{1}^{\prime}(p)=\tau_{1}\left(\phi_{1}(p)\right)$. The left hand side is the Laplace transform of $\widetilde{w}_{1}(p) /\left(p^{2} \widetilde{\tau}_{1}^{\prime}(p)\right)$, which is determined to be the inverse Laplace transform of the right hand side, at least for suitable $g_{1}(t)$.

Rheologically, the consequences of this observation are quite important as it shows that it is not necessary to assume the fading memory associated with the relaxation spectrum is the same as the fading memory of the molecular weight distribution in terms of how both these quantities determine the corresponding relaxation modulus $G_{1}(t)$. In fact, this result holds for all values of the reptation parameter $r$.

INTERPRETATION OF THE SINGLE REPTATION INVERSION FORMULA. As an immediate consequence of (26), one has the following explicit dual formulas for the single reptation components of the molecular weight distribution

$$
w_{1}(m)=\frac{\bar{H}_{1}(m) \tau_{1}^{\prime}(m)}{G_{N}^{0} k_{1}(m) \tau_{1}(m)}, \quad \bar{H}_{1}(m)=\frac{G_{N}^{0} w_{1}(m) k_{1}(m) \tau_{1}(m)}{\tau_{1}^{\prime}(m)} .
$$

Though an interesting expression theoretically, this illustrates, from a practical perspective, the magnitude of the problem involved before an estimate of $w_{1}(m)$ can be obtained. It is necessary to determine, from an appropriate estimate of the relaxation spectrum $H(\tau)$, the single reptation component $H_{1}(\tau)$. Independently, it is necessary to determine the single reptation form of $\tau_{1}(m)$, as well as the form of $k_{1}(m)$.

Setting $k_{1}(m)=1 / m$ and $\tau_{1}(m)=K_{1} m^{\alpha_{1}}$, one obtains

$$
w_{1}(m)=\frac{\alpha_{1} \bar{H}_{1}(m)}{G_{N}^{0}}, \quad \bar{H}_{1}(m)=\frac{G_{N}^{0} w_{1}(m)}{\alpha_{1}},
$$

which correspond, up to constant factors, to the [16] formulas with $\beta=1$.

Rheologically, this result implies that, if the dominant polymer dynamics is single reptation and the relaxation time dependence on the molecular weight is defined by $\tau_{1}(m)=K_{1} m^{\alpha_{1}}$, then the molecular distribution is proportional to the relaxation spectrum after being rescaled by the change of variable from relaxation time to molecular weight. In many ways, this conclusion identifies both the strength and the weakness of the single reptation model of polymer dynamics; namely, it allows one to make some precise conclusions about the associated molecular dynamics at the expense of oversimplifying the nature of the underlying polymer dynamics. 
DOUBLE REPTATION. The situation $r=2$ corresponds to double reptation polymer dynamics. Setting $r=2$ in equation (19), introducing the change of variable $p=1 / \tau_{2}(m)$ and writing $m=\phi_{2}(p)$ for the inverse relation, one finds that

$$
\int_{0}^{\infty} \frac{\widetilde{H}_{2}(p)}{p} \exp \left(-g_{2}(t) p\right) d p=G_{N}^{0}\left[\int_{0}^{\infty} \frac{\widetilde{k}_{2}(p)}{p^{2} \widetilde{\tau}_{2}^{\prime}(p)} \exp \left(-f_{2}(t) p\right) \widetilde{w}_{2}(p) d p\right]^{2}
$$

where $\widetilde{H}_{2}(p)=\widetilde{H}_{2}\left(\phi_{2}(p)\right), \widetilde{\tau}_{2}^{\prime}(p)=\tau_{2}^{\prime}\left(\phi_{2}(p)\right), \widetilde{k}_{2}(p)=k_{2}\left(\phi_{2}(p)\right)$, and $\widetilde{w}_{2}(p)=w_{2}\left(\phi_{2}(p)\right)$.

Applying the Laplace convolution theorem to the right hand side of (29), one obtains

$$
\begin{gathered}
\int_{0}^{\infty} \frac{\widetilde{H}_{2}(p)}{p} \exp \left(-g_{2}(t) p\right) d p=G_{N}^{0} \int_{0}^{\infty} \exp \left(-f_{2}(t) p\right) \\
{\left[\int_{0}^{p} F(q, p-q) \widetilde{w}_{2}(q) \widetilde{w}_{2}(p-q) d q\right] d p} \\
F(q, p-q)=\frac{\tilde{k}_{2}(q) \widetilde{k}_{2}(p-q)}{q^{2}(p-q)^{2} \widetilde{\tau}_{2}^{\prime}(q) \tilde{\tau}_{2}^{\prime}(p-q)} .
\end{gathered}
$$

For given $\widetilde{H}_{2}(p), \widetilde{\tau}_{2}(p), g_{2}(t)$ and $f_{2}(t)$, this last expression uniquely determines

$$
\int_{0}^{p} F(q, p-q) \tilde{w}_{2}(q) \tilde{w}_{2}(p-q) d q=(\Theta * \Theta)(p), \quad \Theta(q)=\frac{\tilde{k}_{2}(q) \tilde{w}_{2}(q)}{q \widetilde{\tau}_{2}^{\prime}(q)}
$$

where the star $(*)$ denotes convolution. This follows by the same argument used above to establish the uniqueness of $\widetilde{w}_{1}(m)$. Now, if $\Theta_{1}$ and $\Theta_{2}$ are the corresponding expressions for two possible $\widetilde{w}_{1}$ and $\widetilde{w}_{2}$, then it follows that

$$
\Theta_{1} * \Theta_{1}=\Theta_{2} * \Theta_{2}
$$

where

$$
\left(\Theta_{1}-\Theta_{2}\right) *\left(\Theta_{1}+\Theta_{2}\right)=0
$$

Since the second factor is clearly non-zero when $\widetilde{k}_{2}(p)>0$, it follows from the Titchmarsh convolution theorem (an elementary proof is given in [8]) that the first factor vanishes, and, hence, that $\widetilde{w}_{1}=\widetilde{w}_{2}$.

On assuming that $f_{2}(t)=g_{2}(t)$, one obtains as a corollary

$$
\frac{\widetilde{H}_{2}(p)}{p}=G_{N}^{0} \int_{0}^{p} F(q, p-q) \widetilde{w}_{2}(q) \widetilde{w}_{2}(p-q) d q
$$

This leaves open the question of the existence of solutions of $(29)$ when $f_{2}(t) \neq g_{2}(t)$. However, one can repeat the argument in the previous section, which was applied to equation (27), to equation (29). 
INTERPRETATION OF THE DOUBLE REPTATION INVERSION FORMULAS. In the special situation where $f_{2}(t)=g_{2}(t)=t, \tau_{2}(m)=K_{2} m^{\alpha_{2}}$ and $k_{2}(m)=1$, the above expression (31) becomes, on setting $p=1 /\left(K_{2} m^{\alpha_{2}}\right)$ and $d p / p=-\alpha_{2} d m / m$,

$$
\begin{aligned}
\int_{0}^{\infty} \frac{\widetilde{H}_{2}(p)}{p} \exp (-t p) d p=\frac{G_{N}^{0}}{\alpha_{2}^{2} K_{2}^{2 / \alpha_{2}}} \int_{0}^{\infty} \exp (-t p) \\
\\
{\left[\int_{0}^{p} \frac{\widetilde{w}_{2}(q) \widetilde{w}_{2}(p-q)}{q^{\left(\alpha_{2}+1\right) / \alpha_{2}}(p-q)^{\left(\alpha_{2}+1\right) / \alpha_{2}}} d q\right] d p }
\end{aligned}
$$

which, by the uniqueness of the Laplace transform, yields

$$
\frac{\tilde{H}_{2}(p)}{p}=\frac{G_{N}^{0}}{\alpha_{2}^{2} K_{2}^{2 / \alpha_{2}}}\left[\int_{0}^{p} \frac{\widetilde{w}_{2}(q) \widetilde{w}_{2}(p-q)}{q^{\left(\alpha_{2}+1\right) / \alpha_{2}}(p-q)^{\left(\alpha_{2}+1\right) / \alpha_{2}}} d q\right]
$$

If the change of variables from $m$ to $p$ is now reversed, one obtains, with $q=1 /\left(K_{2} \bar{m}^{\alpha_{2}}\right)$,

$$
\bar{H}_{2}(m)=\frac{m G_{N}^{0}}{\alpha_{2}} \int_{m}^{\infty} \frac{\bar{m}^{\alpha_{2}+1}}{\left(\bar{m}^{\alpha_{2}}-m^{\alpha_{2}}\right)^{\left(\alpha_{2}+1\right) / \alpha_{2}}} w_{2}(\bar{m}) w_{2}\left(\left[\frac{m \bar{m}}{\left(\bar{m}^{\alpha_{2}}-m^{\alpha_{2}}\right)^{1 / \alpha_{2}}}\right]\right) d \bar{m} .
$$

Since the mapping between relaxation times and their corresponding molecular weights is a proportional relationship, the values of $\bar{H}_{2}(m)$ for large $m$ correspond to the values of $H(\tau)$ for large $\tau$. Consequently, the convolutional structure of the above formula indicates that the contribution of a longer relaxation time $\tau_{\ell}$ to the spectrum depends on the interaction of all the molecules with a molecular weight greater than $m_{\ell}=\left(\tau_{\ell} / K_{2}\right)^{1 / \alpha_{2}}$, which corresponds to the larger molecules with a molecular weight greater than $m_{\ell}$. Interestingly, this formula indicates that the shorter relaxation times depend on a greater range of molecular weight polymer chain interactions than the longer times. Among other things, this shows the deeper aspects of polymer dynamics hidden within their double reptation behaviour.

Triple Reptation. Setting $r=3$ in equation (19), introducing the change of variable $p=1 / \tau_{3}(m)$ and writing $m=\phi_{3}(p)$ for the inverse relation, one finds that

$$
\int_{0}^{\infty} \frac{\tilde{H}_{3}(p)}{p} \exp \left(-g_{3}(t) p\right) d p=G_{N}^{0}\left[\int_{0}^{\infty} \frac{\tilde{k}_{3}(p)}{p^{3} \widetilde{\tau}_{3}^{\prime}(p)} \exp \left(-f_{3}(t) p\right) \widetilde{w}_{3}(p) d p\right]^{3},
$$

where $\widetilde{H}_{3}(p)=\bar{H}_{3}\left(\phi_{3}(p)\right), \widetilde{\tau}_{3}^{\prime}(p)=\tau_{3}^{\prime}\left(\phi_{3}(p)\right), \widetilde{k}_{3}(p)=k_{3}\left(\phi_{3}(p)\right)$, and $\widetilde{w}_{3}(p)=w_{3}\left(\phi_{3}(p)\right)$.

The Laplace convolution theorem allows the cube to be replaced by the transform of a triple convolution, which brings one back to a situation similar to that for double reptation.

Higher LEVELS OF REPTATION. The process of constructing analytic relationships for higher and higher levels of reptation continues inductively. 


\section{REFERENCES}

[1] F. Akyildiz, R.S. Jones and K. Walters, 'On the spring-dashpot representation of linear viscoelastic behaviour', Rheol. Acta 29 (1990), 482-484.

[2] R.S. Anderssen and R.J. Loy, 'On the scaling of molecular weight distribution functionals', J. Rheol. 45 (2001), 891-901.

[3] R.S. Anderssen and D.W. Mead, 'Theoretical derivation of molecular weight scaling for rheological parameters', J. Non-Newtonian Fluid Mech. 76 (1998), 299-306.

[4] R.S. Anderssen and M. Westcott, 'The molecular weight distribution problem and reptation mixing rules', ANZIAM J. 42 (2000), 26-40.

[5] A N. Beris and B.J. Edwards, 'On the admissability criteria for linear viscoelasticity kernels', Rheol. Acta 32 (1993), 505-510.

[6] L. Boltzmann, 'Zur Theorie der elastischen Nachwirkung', Ann. Phys. Chem. 7 (1876), 624-657.

[7] W. A. Day, 'On monotonicity of the relaxation functions of viscoelastic materials', Proc. Camb. Philos. Soc. 67 (1970), 503-508.

[8] R. Doss, 'An elementary proof of 'Titchmarsh's convolution theorem', Proc. Amer. Math. Soc. 104 (1988), 181-184.

[9] M. Fabrizio and A. Morro, Mathematical problems in linear viscoelasticity, SIAM Studies in Applied Mathematics 12 (SIAM, Philadelphia, 1992).

[10] W. Feller, An introduction to probability theory and its applications 2 (John Wiley and Sons, New York, 1971).

[11] A. Z. Grinshpan, M.E.H. Ismail and.D.L. Milligan, 'Complete monotonicity and diesel fuel spray', Math. Intelligencer 22 (2000), 43-53.

[12] R.G. Larson, Constitutive equations for polymer melts and solutions (Butterworths, Boston, 1988).

[13] F. Léonardi, J.-C. Majesté, A. Allal and G. Marin, 'Rheological models based on the double reptation mixing rule: The effect of a polydisperse environment', J. Rheol. 44 (2000), 675-692.

[14] T.C.B. McLeish and R.G. Larson, 'Molecular constitutive equations for a class of branched polymers: The pom-pom polymer,', J. Rheol. 42 (1998), 81-110.

[15] D.W. Mead, 'Determination of molecular weight distributions of linear flexible polymers from linear viscoelastic material functions', J. Rheol. 38 (1994), 1797-1827.

[16] W. Thimm, C. Friedrich, M. Marth and J. Honerkamp, 'An analytical relation between relaxation time spectrum and molecular weight distribution', J. Rheol. 43 (1999), $1663-1672$.

[17] W. Thimm, C. Friedrich, M. Marth and J. Honerkamp, 'On the Rouse spectrum and the determination of the molecular weight distribution', J. Rheol. 44 (2000), 429-438.

[18] D.V. Widder, The Laplace transform (Princeton University Press, Princeton, 1946).

[19] S. Wu, 'Characterization of polymer molecular weight distribution by transient viscoelasticity: Polytetrafluoroethylenes', Poly. Eng. Sci. 28 (1989), 538-543.

CSIRO Mathematical and Information Sciences GPO Box 664

Canberra ACT 2601

Australia
Mathematics Department

School of Mathematical Sciences

Australian National University

Canberra ACT 0200

Australia 